\title{
A Microwave Scattering Model for Simulating the C-Band SAR Backscatter of Wheat Canopy
}

\author{
Wenjia Yan ${ }^{1}$, Yuan Zhang ${ }^{1,2, *}$, Tianpeng Yang ${ }^{1}$, Xiaohui Liu ${ }^{3}$ \\ ${ }^{1}$ Key Laboratory of Geographic Information Science (Ministry of Education), East China Normal University, Shanghai, China \\ ${ }^{2}$ Institute of Eco-Chongming, East China Normal University, Shanghai, China \\ ${ }^{3}$ Key Laboratory of Wetland Ecology and Environment, Northeast Institute of Geography and Agroecology, Chinese Academy of Sciences, \\ Changchun, China
}

\section{Email address}

wenjiayan1002@163.com (Wenjia Yan),yzhang@geo.ecnu.edu.cn (Yuan Zhang),yangtianpeng1219@163.com (Tianpeng Yang), xhliu1978@yahoo.com (Xiaohui Liu)

*Corresponding author

\section{To cite this article:}

Wenjia Yan, Yuan Zhang, Tianpeng Yang, Xiaohui Liu. A Microwave Scattering Model for Simulating the C-Band SAR Backscatter of Wheat Canopy. American Journal of Remote Sensing. Vol. 7, No. 1, 2019, pp. 13-24. doi: 10.11648/j.ajrs.20190701.13

Received: July 11, 2019; Accepted: July 31, 2019; Published: September 20, 2019

\begin{abstract}
Accurate simulation of microwave scattering characteristics of wheat canopy can provide valuable insights into the scattering mechanisms of wheat crops. In this study, a wheat canopy scattering model (WCSM) was developed on a basis of first-order microwave radiative transfer equation. Several WCSM inputs, including wheat canopy and soil parameters, were measured in situ at the time (or near the time) of the satellite observation. The backscattering coefficients of wheat fields were then simulated at various incident angles and polarization modes. Four C-band quad-polarized (Radarsat-2 and Gaofen-3) SAR data were used to evaluate the WCSM performance in four key growth stages of winter wheat from stem elongation to ripening in 2017. Results showed that the WCSM simulated backscattering coefficients of wheat fields with error lower than $1.8 \mathrm{~dB}$. This study demonstrates that the proposed WCSM is effective in characterizing the C-band backscatter features of wheat crops for various growth phases. It also indicated that the operational potential of C-band satellite SAR systems such as the Radarsat2 and the China Gaofen-3 SAR in monitoring wheat growth for food safety in important agricultural regions.
\end{abstract}

Keywords: Wheat Canopy, Wheat Canopy Scattering Model (WCSM), Synthetic Aperture Radar (SAR), C-band, Backscatter, Simulation

\section{Introduction}

Wheat is one of the staple food crops in many regions around the world [1]. In China, the demand for wheat grain is increasingly rising with the ongoing population growth and rapid urbanization. Reliable wheat monitoring is thus crucial for sustainable development of agriculture and food security. As an active remote sensing technology, synthetic aperture radar (SAR) is well suited to long-term monitoring wheat over large areas as its all-weather and day to night imaging capability [2]. This has been proved by a large number of studies using a stack of radar images for mapping crop planting area [3-5] and monitoring crop growth [6-8]. Radar wave at C-band is able to penetrate into wheat canopy, hence the return signals that often serves as an indicator of crop healthiness provide structural information of crop canopy and underlying grounds. Existing study on polarimetric features showed that, for those vertically oriented crops such as wheat, horizontally polarized microwaves tend to penetrate the crop canopy to a greater extent than vertically polarized ones at steep incidence angles. Cross-polarized radar returns are sensitive to crop structure within the total canopy volume [9]. Therefore, quantitatively characterizing physical scattering mechanisms of wheat canopy plays a role in linking biophysical properties of wheat plants to radar signals.

There have been an increasing number of ground-based scatterometer or spaceborne radar experiments to measure the responses of radar backscatter to wheat structural properties and sensor configurations [10,11]. A study stated that C-band $\mathrm{VV}$ signals at $23^{\circ}$ were mostly sensitive to wheat biomass, 
while the $\mathrm{HH}$ had a strong correlation with soil water content [12]. Jia et al. (2013) found that radar HH signals and cross polarizations $(\mathrm{HV}, \mathrm{VH})$ in L-band were more correlated with biomass and LAI in comparison with S-, C- and X-band. At the same time, this observation study also revealed the insensitivity of $\mathrm{C}$-band microwave signals at linear polarizations to soil moisture [13]. Otherwise for the spaceborne observations, a sensitivity analysis conducted by Balenzano et al. (2011) showed that the C-band SAR signal was considerably influenced by the canopy structure and the phenological stage of wheat crops, whereas the L-band backscatter was insensitive to the wheat canopy and $\mathrm{HH}$ polarization was better suited to soil moisture monitoring [14]. Fontanelli et al. (2013) reported that radar signals of X-band SAR had a rather high sensitivity to wheat LAI at both $\mathrm{HH}$ and VV polarizations [15]. A negative correlation between HV polarization and wheat height was found from Radarsat-2 SAR data during the elongation stage [16]. A recent study indicated that the L-band radar signal in $\mathrm{HH}$ polarization can penetrate more well-developed wheat canopy, and is more sensitive to soil surface moisture than C-band in both $\mathrm{VV}$ and $\mathrm{VH}$ polarizations [17]. These above studies indicated that there is a need to quantitatively interpret the $\mathrm{C}$-band microwave scattering mechanisms of wheat fields with respect to different radar configurations, phenological phases and environment conditions.

Until now, many modelling studies have been substantially conducted to quantify the radar responses of various crop canopies. Given the simplicity and physical background, semi-empirical models based on original water cloud model (WCM) [18] were often applied to wheat canopy scattering simulation [19-22]. To further interpret radar signals of crop canopies, physical models driven by datasets measured at crop fields were developed to better explain scattering mechanisms in the growing period. A simplified Michigan microwave canopy scattering model omitting trunk components was developed to simulate scatter coefficients of wheat [23]. A higher simulation precision was achieved for $\mathrm{HH}$ polarization than $\mathrm{VV}$ at $\mathrm{L}$ and $\mathrm{C}$ bands over a growing season. A multiple-scattering model [24] was refined for wheat. The internal field of the hollow infinite cylinder in Della Vecchia et al (2006) was computed under the approximation of infinite length [25]. However, the cylinder had finite length since a truncation was applied, similarly to the case in [26]. It was demonstrated that wheat backscatter coefficients were significantly influenced by the stem hollowness and a more detailed characterization of the crop geometry might be conducive to improve the model accuracy. A second order radiative transfer model was also established to simulate the backscatter of wheat canopy [27]. Simulation results showed that the second-order scattering terms had a minor contribution to the total canopy backscatter with regard to the C-band full polarization data. Meanwhile, a phase-coherent scattering model was proposed to explain the co-polarized scattering mechanism of wheat fields [28]. However, this model did not predict an effective backscatter for the stages with grain heads fully emerged [29]. After that, a coherent electromagnetic model was presented to compare C-band scattering of wheat against that of sunflower [30]. In the case of wheat, double scattering of soil-stalk was the most considerable contributor to the total canopy. In addition, a multiple scattering model was developed on a basis of the Foldy-Lax multiple-scattering equation, wherein the model only provided good estimates in VV polarization of wheat backscattering coefficients [31, 32]. In the abovementioned modelling studies, ear contributions to the total scattering response were often neglected or the surface scattering of ground was simplified. However, the wheat backscatter is directly determined by the canopy architecture and geometric/physical properties of plant constituents. In our recent pilot study, a microwave scattering model for rice was modified to simulate the multitemporal C-band Sentinel-1 SAR responses of wheat canopy [33]. Simulation results showed the model was effective in dual-polarized data ( $\mathrm{VH}$ and $\mathrm{VV}$ ) at incidence angle of $43.15^{\circ}$, while a relatively large error of $(\sim 3 \mathrm{~dB})$ was found with an incidence angle of $32.12^{\circ}$. Therefore form the perspective of operational monitoring of wheat growth in the future, the backscatter mechanisms of wheat canopy requires to be further quantified precisely to achieve a better simulation.

The objective of this study is to interpret the C-band SAR features of wheat fields and improve the understanding for microwave scattering mechanisms of wheat canopy. A wheat canopy scattering model (WCSM) was proposed to characterize backscattering characteristics with changing wheat canopies at different growth stages. Four C-band Radarsat-2 and Gaofen-3 quad-polarized SAR images covering a major winter-wheat production area in North China were acquired corresponding to four key growth stages. They served as reference data to evaluate applicability and performance of the WCSM.

\section{Dataset and Methodology}

\subsection{Study Area}

The study area is situated at Wuji county of the Huabei Plain (Centered at $114.13^{\circ} \mathrm{E}, 38.01^{\circ} \mathrm{N}$ ) (Figure 1), which is one of major wheat production areas in North China. With a typical semi-arid monsoon climate, rainfall occurring mostly during summer (June through August), 3 to 4 irrigations are practiced during the entire growing season from middle October to early June of the subsequent year. The winter wheat experiences three major developing stages in one growth cycle, i.e. vegetative phase (mid-October to midApril next year), reproductive phase (late April to mid-May), ripening phase (late May to early June). The flat terrain, vast field size and significant phenological characters of wheat canopies provide highly favorable conditions for field survey for wheat parameter measurements and remote sensing applications in wheat growth monitoring. 


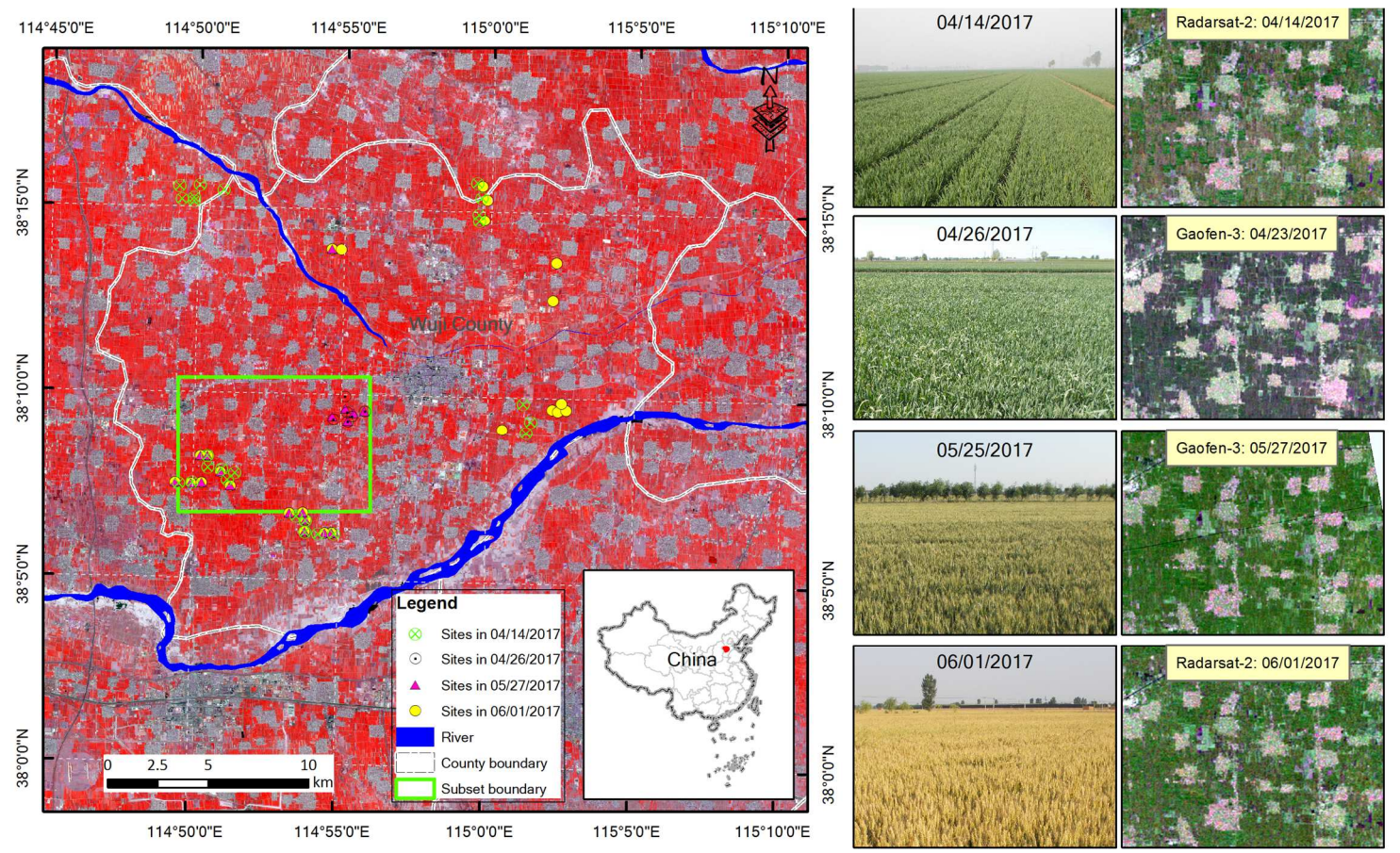

Figure 1. Study area and field sites in 2017. Where, SAR data subsets with band composition of $H H(R), V H(G)$ and VV (B) and corresponding field photographs were respectively shown in the right hand.

\subsection{Data Set}

\subsubsection{Satellite SAR Data}

A total of $4 \mathrm{C}$-band multi-temporal quad-polarization images $(\mathrm{HH}+\mathrm{HV}+\mathrm{VH}+\mathrm{VV})$ were acquired over the study area in 2017 as shown in Figure 1. They were remotely sensed by two sensors, Radarsat-2 and Gaofen-3 SAR at the elongating (April 14), booting (April 23), milking (May 27) and ripening stages (June 1) of winter-wheat, respectively (Table 1). For Radatsat-2 imagery, multilooking (1 and 2 of look numbers for range and azimuth direction), radiometric calibration, speckle noises suppression (Gamma-MAP filter of $5 \times 5$ window) and terrain correction ( $30 \mathrm{~m}$ resolution of SRTM DEM data) were processed by the SNAP V6.0 toolbox (http://step.esa.int/main/download/) step by step. The same processing approaches were utilized for the Gaofen-3 SAR data. In addition, all the four backscattering coefficient $\left(\sigma^{\circ}\right)$ maps were resampled to a spatial resolution of $10.0 \mathrm{~m}$ using the nearest neighbor algorithm, and geometrically coregistered to a projection of the Universal Transverse Mercator system Zone 50, WGS 84. They were separately taken as validation data sets to assess the simulation accuracy of wheat canopy scattering model at each growth stages.

Table 1. Parameters of SAR data for WCSM validation.

\begin{tabular}{|c|c|c|c|c|}
\hline Satellite SAR & Incident angle & Original pixel spacing & Acquisition date & Growth stages of wheat \\
\hline \multirow{2}{*}{ Radarsat-2 } & $23.54^{\circ}$ & $4.73 \mathrm{~m} \times 4.97 \mathrm{~m}$ & April 14 & Elongating \\
\hline & $23.54^{\circ}$ & $4.73 \mathrm{~m} \times 4.97 \mathrm{~m}$ & June 1 & Ripening \\
\hline Gaofen-3 & $38.00^{\circ}$ & $2.25 \mathrm{~m} \times 5.04 \mathrm{~m}$ & April 23 & Booting \\
\hline
\end{tabular}

\subsubsection{Field Survey Data}

Ground truth data were collected on April 14, April 26, May 25 and June 1, 2017 for wheat biophysical parameters acquisition. The first field campaign on April 14 was rightly in the elongating stage. In this critical stage of winter-wheat growth, irrigation was normally required to meet the large demand of water. In booting stage (April 26), wheat plants reached the growth peak in vegetative phase with the longest leaves and thicker stems. For the last two field trips, there was almost no increase of the wheat canopy height at grain milking and ripening stages. Wheat components became turning yellow and drying with gradual decrease in water content until harvest.
A total of 75 sampling sites were observed from the whole study area (Figure 1). Wheat structural parameters measured at each site consisted of ear size (length and diameter), leaf size (length, width and thickness) and stem size (length and diameter), number of leaves per plant, number density of wheat plants and canopy height of wheat (Table 2). Wheat plants were sampled and the gravimetric moisture fraction of each wheat constituents (ears, leaves and stems) were calculated by oven drying method. Complex dielectric constants of wheat layers were calculated separately based on the Debye-Cole dual-dispersion model [34]. The average of 4 measurements within a sampling plot was calculated to represent in situ data for one observation site. Soil volumetric water content fraction (WCF) at a depth of $7 \mathrm{~cm}$ was 
collected at field using a portable Takeme-10 soil moisture/temperature meter, which was used to compute the complex dielectric constants by an empirical model [35]. In addition, 3 wheat plants were randomly selected in each sampling site for in situ measurements. A total of 266 leaf insertion angles (the angle between long axis of leaf and wheat stem) were measured at the same time.

Table 2. Ground truth data of wheat biophysical parameters used in model simulation.

\begin{tabular}{|c|c|c|c|c|c|}
\hline \multirow{2}{*}{ Items } & \multirow{2}{*}{ Parameters } & \multicolumn{4}{|c|}{ Parameter ranges for different growth stages } \\
\hline & & Elongating & Booting & Milking & Ripening \\
\hline \multirow{2}{*}{ Canopy } & Height $(\mathrm{cm})$ & $31.7-47.0$ & $55.7-58.7$ & $75.0-83.0$ & $64.0-83.3$ \\
\hline & Number density $\left(\# / \mathrm{m}^{2}\right)$ & $940-1215$ & $940-1215$ & $752-976$ & $624-928$ \\
\hline \multirow{3}{*}{ Ear } & Length $(\mathrm{cm})$ & - & - & $7.5-8.7$ & $5.6-8.4$ \\
\hline & Diameter $(\mathrm{cm})$ & - & - & $0.95-1.15$ & $0.90-1.30$ \\
\hline & Gravimetric $\mathrm{WCF}^{*}$ & - & - & $0.531-0.600$ & $0.409-0.539$ \\
\hline \multirow{4}{*}{ Leaf } & Length $(\mathrm{cm})$ & $13.4-19.3$ & $16.8-20.0$ & $15.7-18.6$ & $12.8-21.5$ \\
\hline & Width $(\mathrm{cm})$ & $0.93-1.42$ & $1.09-1.33$ & $1.16-1.34$ & $0.54-1.15$ \\
\hline & Thickness $(\mathrm{cm})$ & $0.020-0.028$ & $0.018-0.022$ & $0.017-0.018$ & $0.010-0.016$ \\
\hline & Gravimetric WCF & $0.795-0.873$ & $0.732-0.787$ & $0.700-0.762$ & $0.286-0.771$ \\
\hline \multirow{3}{*}{ Stem } & Length (cm) & $15.43-30.63$ & $36.20-45.13$ & $60.0-69.0$ & $53.7-72.0$ \\
\hline & Diameter $(\mathrm{cm})$ & $0.302-0.415$ & $0.305-0.355$ & $0.308-0.343$ & $0.257-0.366$ \\
\hline & Gravimetric WCF & $0.823-0.903$ & $0.804-0.876$ & $0.713-0.775$ & $0.590-0.761$ \\
\hline \multirow{3}{*}{ Ground surfaces } & rms height (cm) & 0.8 & 0.8 & 0.5 & 0.5 \\
\hline & Correlation length $(\mathrm{cm})$ & 6.0 & 6.0 & 6.0 & 6.0 \\
\hline & Volumetric WCF & $0.115-0.316$ & $0.134-0.217$ & $0.145-0.203$ & $0.117-0.208$ \\
\hline
\end{tabular}

* WCF: water content fraction.

In this study, all the measured and derived parameters on 75 sampling sites were compiled as inputs for the WCSM. Five randomly selected sites out of the 75 total were used for WCSM parameterization, while the remaining 70 sites for WCSM validation (i.e. 20 on April 14, 6 on April 26, 19 on May 25 and 25 on June 1, 2017).

\subsection{Wheat Canopy Scattering Model}

A wheat canopy scattering model based on first-order radiative transfer equation was proposed to formulate the scattering mechanisms of wheat crops. Wheat canopy were regarded as a double-layered dielectric medium over a rough soil surface (Figure 2). Ears were treated as randomly oriented short dielectric cylinders, and stems as vertical cylinders with finite length. The stems were assumed to be almost erectophile, and the ears were slightly plagiophile but more vertical inclined in morphology. Leaves were simulated as long and thin elliptic dielectric discs. The upper layer (or layer 1) consisted of short cylindrical ears and small part of elliptic disk-shaped leaves (i.e. $20 \%$ of total), while the lower layer (or layer 2) was a combination of the rest part of leaves (i.e. $80 \%$ of total) and long and slim cylindrical stems. The scattering intensity of each scatter was attenuated by additive scatterers in the same layer and/or adjacent layer. The upper layer were not considered before the heading stage. Surface scattering from rough soil surfaces were calculated by the advanced integral equation method (AIEM) [36] that was incorporated into the WCSM.

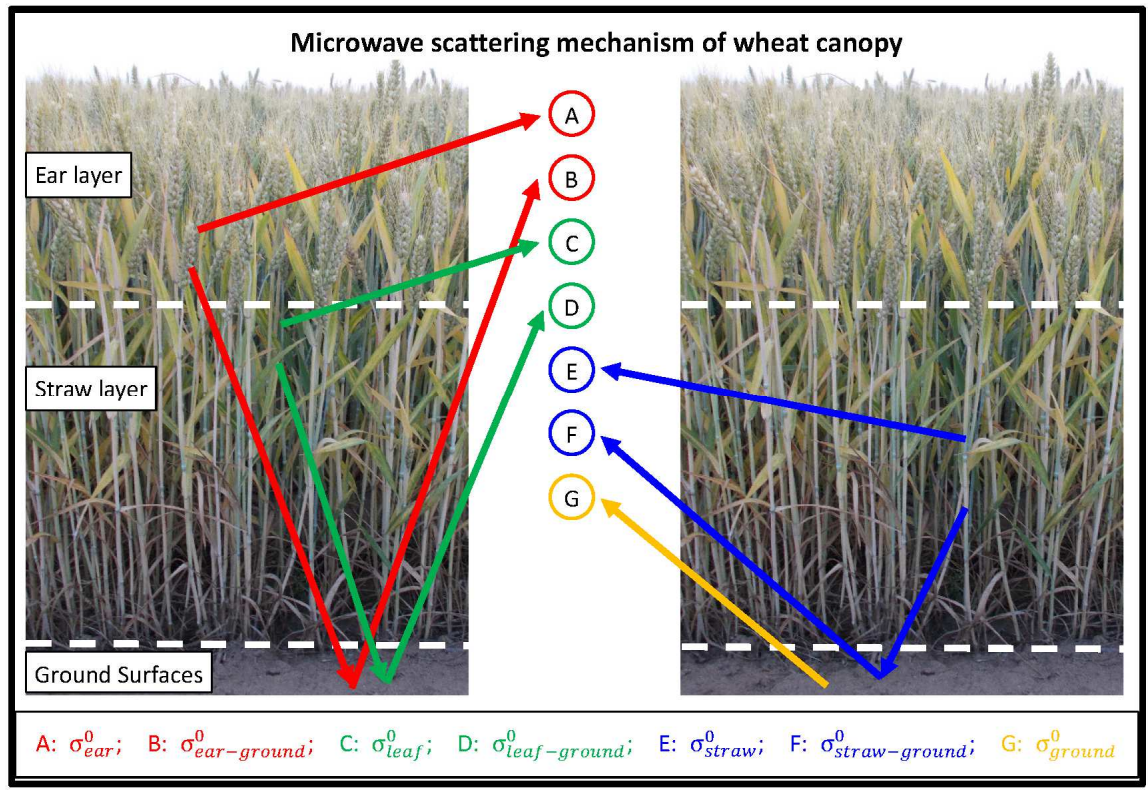

Figure 2. Microwave scattering mechanisms of wheat canopy. 
In WCSM, total backscatter intensity ( $\sigma_{\text {total }}$ in power unit) was a linear composition of volume scattering of each constituents ( $\sigma_{\text {ear }}, \sigma_{\text {leaf }}$ and $\sigma_{\text {stem }}$ ), its double-bounce scattering with ground surface ( $\sigma_{\text {ear-ground }}, \sigma_{\text {leaf-ground }}$ and $\left.\sigma_{\text {stem-ground }}\right)$ and surface scattering of ground $\left(\sigma_{\text {ground }}\right)$ :

$$
\begin{gathered}
\sigma_{\text {total }}=\sigma_{\text {ground }}+\sigma_{\text {ear }}+\sigma_{\text {ear } \leftrightarrow \text { ground }}+\sigma_{\text {leaf }}+ \\
\sigma_{\text {leaf } \leftrightarrow \text { ground }}+\sigma_{\text {stem }}+\sigma_{\text {stem } \leftrightarrow \text { ground }}
\end{gathered}
$$

\subsubsection{Ear Scattering and Ear-ground Double-bounce Scattering}

Ears were only considered from the heading stage to harvest. An ear was represented as a short cylinder with finite length. In (1), the ear volume scatter (component A in Figure 2: $\left.\sigma_{\text {ear }}\right)$ is an additive contribution from all the ears in the upper layer [37]. A first-order solution to the radiative transfer equation, can be expressed as:

$$
\sigma_{e a r}=4 \pi \cdot Q_{p q}^{e a r} \cdot \frac{1-L_{1 p} L_{1 q}}{\left(k_{1 p}+k_{1 q}\right) \cdot \sec \theta_{i}}
$$

where $Q_{p q}^{\text {ear }}(p, q=\mathrm{H}$ or $\mathrm{V}$ polarization) represents backscattering cross-section per unit volume of ears within the upper layer; $L_{n}$ is the polarized attenuation factor integrated over the depth of the corresponding layer $(n=1,2$ where 1 for ear layer and 2 for stalk layer), and $k_{1}$ is the extinction coefficient for cylinders [26] and elliptic disks [38] in the upper layer; $\theta_{i}$ is the incident angle of the incoming radar signal.

When computing the interactions between scatterers from different layers with ground surfaces, the surface was assumed to be slightly rough to simplify the radiative transfer equation. The ear-ground double-bounce scattering $\sigma_{\text {ear } \leftrightarrow \text { ground }}$ (component B in Figure 2) in (1) can be described as:

$$
\sigma_{\text {ear } \leftrightarrow \text { ground }}=\sigma_{\text {ear } \rightarrow \text { ground }}+\sigma_{\text {ground } \rightarrow \text { ear }}
$$

where

$$
\begin{gathered}
4 \pi \cos \theta_{i} L_{1 p} L_{2 p}^{2} R_{p p} e^{-4 k^{2} s^{2} \cos ^{2} \theta_{i}} Q_{p q}^{e a r} \frac{L_{1 p}-L_{1 q}}{k_{1 q}-k_{1 p}} \\
4 \pi \cos \theta_{i} L_{1 q} L_{2 q}^{2} R_{q q} e^{-4 k^{2} s^{2} \cos ^{2} \theta_{i}} Q_{p q}^{\text {ear }} \frac{L_{1 p}-L_{1 q}}{k_{1 q}-k_{1 p}}
\end{gathered}
$$

where $R_{p p}$ and $R_{q q}$ are the Fresnel reflectivity of ground surface in $p$ or $q$ polarization, respectively; $k$ is the wave number; $s$ is the rms height of the ground surface.

The unit volume scattered intensity of ears in (2), (4), and (5), $Q_{p q}^{e a r}$ was calculated as:

$$
Q_{p q}^{e a r}=N_{e a r}\left\langle\left|F_{p q}^{e a r}\right|^{2}\right\rangle
$$

where $N_{e a r}$ is the number of ears per unit volume, and $F_{p q}^{e a r}$ is the scattering amplitude matrix for a single ear in the upper layer [26]. The ensemble average \langle\rangle represents the statistical average of scattering amplitude tensor for all ears.

\subsubsection{Leaf Scattering and Leaf-ground Double-bounce Scattering}

Wheat leaves were represented as long and thin elliptic disks with three semi-axis of length, width and thickness. Leaf volume scattering $\left(\sigma_{\text {leaf }}\right)$ and its double-bounce scattering ( $\sigma_{\text {leaf } \leftrightarrow \text { ground }}$ ) were contributed by all of the leaves within the layer 1 and the layer 2 (components $C$ and $\mathrm{D}$ in Figure 2). They were computed as indicated below:

$$
\begin{gathered}
\sigma_{\text {leaf }}=\sigma_{\text {leaf } 1}+\sigma_{\text {leaf } 2} \\
\sigma_{\text {leaf } \leftrightarrow \text { ground }}=\sigma_{\text {leaf } 1 \leftrightarrow \text { ground }}+\sigma_{\text {leaf } 2 \leftrightarrow \text { ground }}
\end{gathered}
$$

where

$$
\begin{gathered}
\sigma_{\text {leaf } 1}=4 \pi Q_{p q}^{\text {leaf } 1} \frac{1-L_{1 p} L_{1 q}}{\left(k_{1 p}+k_{1 q}\right) \sec \theta_{i}} \\
\sigma_{\text {leaf } 2}=4 \pi Q_{p q}^{\text {leaf } 2} \frac{1-L_{2 p} \cdot L_{2 q}}{\left(k_{2 p}+k_{2 q}\right) \sec \theta_{i}} L_{1 p} L_{1 q}
\end{gathered}
$$

where $Q_{p q}^{\text {leaf } 1}$ and $Q_{p q}^{\text {leaf } 2}$ in equations (9) and (10) represent backscattering cross-section from leaves per unit volume within the upper layer and lower layer, respectively. Conversely, $Q_{p q}^{\text {leaf } 1}$ and $Q_{p q}^{\text {leaf } 2}$ in equations (11) and (12) is bistatic scattering cross-section per unit volume, respectively. $k_{2}$ is the extinction coefficient of the lower layer.

The unit volume scattered intensity of leaves in (9) through (12), $Q_{p q}^{\text {leaf } 1}$ and $Q_{p q}^{\text {leaf2 }}$ were both computed as:

$$
Q_{p q}^{\text {leaf }}=N_{\text {leaf }}\left\langle\left|F_{p q}^{\text {leaf }}\right|^{2}\right\rangle
$$

where $N_{\text {leaf }}$ is the number density of leaves within the corresponding layer, and $F_{p q}^{\text {leaf }}$ is the scattering amplitude matrix for a leaf [38].

\subsubsection{Stem Scattering and Stem-ground Double-bounce Scattering}

Similarly to the case of ear, stem was simulated as thin finite-length cylinder. The descriptions for the volume scattering of stem $\left(\sigma_{\text {stem }}\right)$ and its interaction with the ground surface $\left(\sigma_{\text {stem } \leftrightarrow \text { ground }}\right)$ are similar to that in (10) and (12) except for the scattering phase matrix [37]. Wherein, $\sigma_{\text {stem }}$ (component E in Figure 2) was described as:

$$
\sigma_{\text {stem }}=4 \pi Q_{p q}^{\text {stem }} \frac{1-L_{2 p} \cdot L_{2 q}}{\left(k_{2 p}+k_{2 q}\right) \sec \theta_{i}} L_{1 p} L_{1 q}
$$


where $Q_{p q}^{\text {stem }}$ represents backscattering cross-section from stems per unit volume within the lower layer (leaves + stems) at $p$ and $q$ polarization.

As similar to (3), the stem-ground double-bounce scattering included two separate terms (component $\mathrm{F}$ in
Figure 2):

$$
\sigma_{\text {stem } \leftrightarrow \text { ground }}=\sigma_{\text {stem } \rightarrow \text { ground }}+\sigma_{\text {ground } \rightarrow \text { stem }}
$$

where,

$$
\begin{aligned}
& \sigma_{\text {stem } \rightarrow \text { ground }}=4 \pi \cos \theta_{i} L_{1 p} L_{1 q} R_{p p} e^{-4 k^{2} s^{2} \cos ^{2} \theta_{i}} Q_{p q}^{\text {stem }} \frac{L_{2 p}-L_{2 q}}{k_{2 q}-k_{2 p}} L_{2 p} \\
& \sigma_{\text {ground } \rightarrow \text { stem }}=4 \pi \cos \theta_{i} L_{1 q} L_{1 p} R_{q q} e^{-4 k^{2} s^{2} \cos ^{2} \theta_{i}} Q_{p q}^{\text {stem }} \frac{L_{2 p}-L_{2 q}}{k_{2 q}-k_{2 p}} L_{2 q}
\end{aligned}
$$

The unit volume scattered intensity of stems in (14), (16), and (17), $Q_{p q}^{\text {stem }}$ was written as:

$$
Q_{p q}^{\text {stem }}=N_{\text {stem }}\left\langle\left|F_{p q}^{\text {stem }}\right|^{2}\right\rangle
$$

where $N_{\text {stem }}$ is the number of stems per unit volume, and $F_{p q}^{\text {stem }}$ is the scattering amplitude matrix for a single stem in the lower layer.

\subsubsection{Ground Surface Scattering}

Ground surface backscattering depends on its dielectric properties, surface roughness, and attenuation from the wheat canopies above the surface. The exponential auto-correlation function was selected for slightly rough soil surfaces under local agricultural practices. The backscatter of ground surface after canopy attenuation was calculated as (component $\mathrm{G}$ in Figure 2):

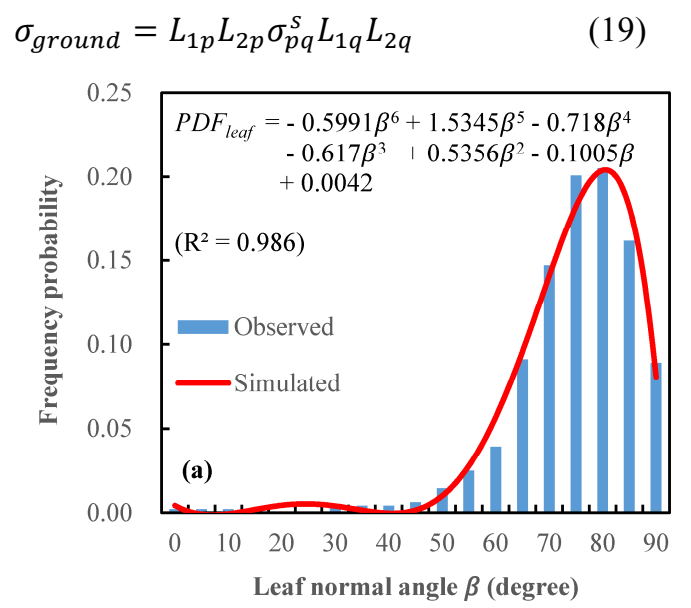

where $\sigma_{p q}^{s}$ represents the surface scattering matrix, which was calculated by the surface scattering model AIEM [36].

\subsubsection{Probability Distribution Function of Wheat Constituents}

The orientation of wheat canopy scatterers was separately simulated by a probability distribution functions (PDFs) (Figure 3). For cylindrical ears and stems, the inclination angle $(\beta)$ represents the angle between vertical and the cylinder axis. The stems were assumed to be almost erectophile, and the ears were slightly plagiophile but more vertical inclined in morphology (Figure $3 b$ ). Leaf inclination angle $(\beta)$ signifies the angle between vertical and the normal vector of the elliptic disk. It was then calculated through an equation: $\beta=90^{\circ}$ - leaf insertion angle. The PDF of leave angles was then formulated by a polynomial regression (Figure 3a). Normalization was ultimately employed to all PDFs within the WCSM.

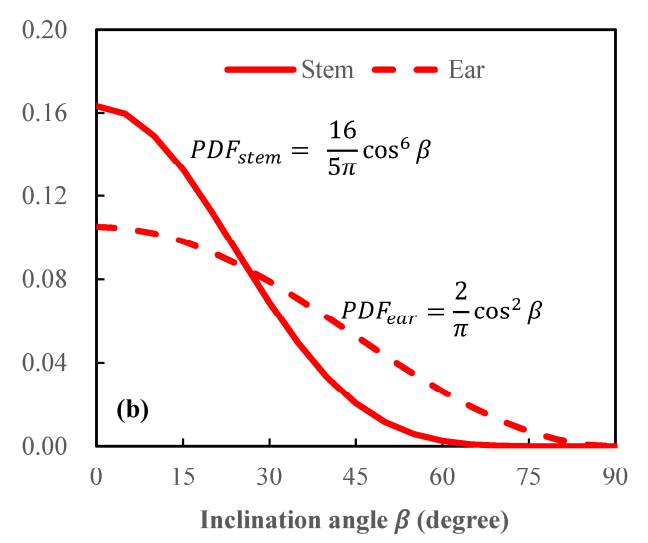

Figure 3. The probability distribution function (PDF) for wheat leaves (a), and wheat ears and stems (b).

\section{Result}

\subsection{Validation of the Simulated C-band Backscatter of Wheat Canopy}

The developed WCSM was validated by comparing the simulated backscattering coefficients $\left(\sigma^{0}\right)$ against the observed ones from SAR images acquired at four growth stages. For each growth stage, 6 parameters including wheat plants and ground surfaces were respectively assigned to a constant value by averaging all the measurements at sampling sites, i.e. stem diameter, leaf width and thickness, roughness (rms height and correlation length) and temperature of soil surfaces. The WCSM was then driven by inputs as listed in Table 2, and total backscattering coefficients of wheat canopy were consequently simulated for 4 growth stages.

For each polarization at 70 validation sites, the scatterplot of simulated against the observed from SAR data is mostly scattered along the 1: 1 line (Figure 4). The WCSM simulated more accurate backscattering coefficients for the cross polarization than co-polarized ones, with the RMSE of $1.37 \mathrm{~dB}$ and MAE of $1.15 \mathrm{~dB}$. The sampling sites with large discrepancies marked with ellipses are presented explicitly in Figure 4. In general, the simulated results agree well with those from SAR image at ripening stage (June 1) with error 
of $<1.6 \mathrm{~dB}$. However, large deviation occurs in one exceptional site with underestimation of $2.8 \mathrm{~dB}$ in $\mathrm{HH}$ and $2.6 \mathrm{~dB}$ in $\mathrm{VH}$ (grey dashed-line circles or ellipse in Figures. $4 \mathrm{a}, \mathrm{c})$. These errors are probably attributed to the radiometric error of the Radarsat-2 image itself. By comparing those pixels having larger error with adjacent pixels, it is found that several abnormal bright points appear in SAR images, corresponding to large backscattering coefficients. In addition, these underestimations could be partially due to neglecting leaf bending in WCSM, as well as image processing algorithms such as the resampled pixel size (10 $\mathrm{m})$ and noise filtering window (5 pixels $\times 5$ pixels). For the elongating (April 14) stage and the booting stage (April 23), simulated values at $\mathrm{HH}$ polarization are $4.0 \mathrm{~dB}$ higher than observed values (Figure 4a). The evident feature for these cases results from the view directions of radar sensors that are almost parallel to the row direction of wheat fields. Conversely, for two sampling sites on April 23, the simulated backscattering coefficients in $\mathrm{HH}$ and $\mathrm{VV}$ are significantly lower ( 3 to $8 \mathrm{~dB}$ ) than the SAR observed ones (Figures $4 \mathrm{a}$, b). After precise examination, the two sites are rightly located in wheat fields with row direction perpendicular to the incident radar waves. Such cases have been reported [31] that azimuth angle between field row and radar direction makes a considerable impact on the attenuation of the wheat canopy.

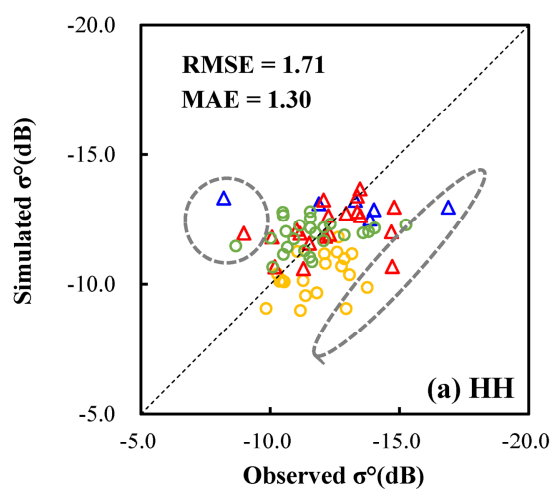

- Apr. 14

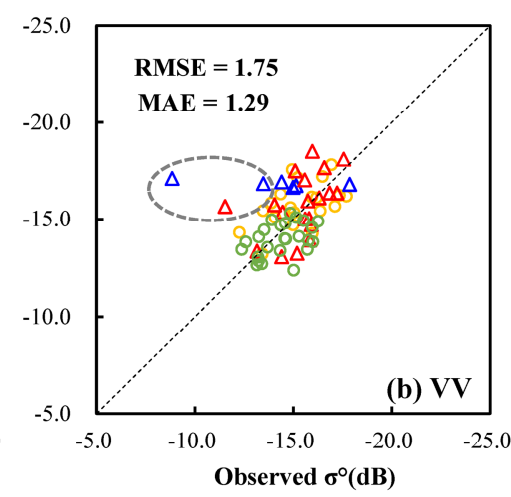

$\Delta$ May. 27

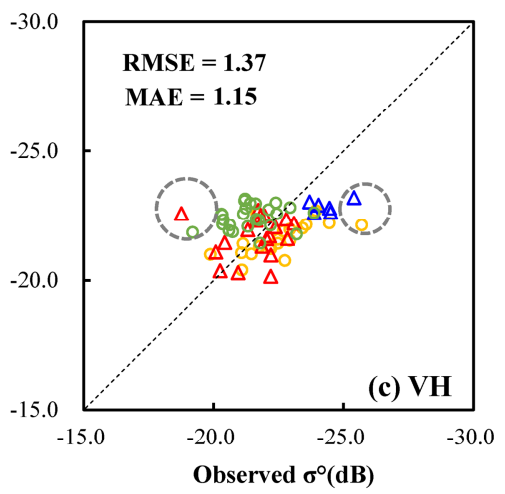

○ Jun. 1

Figure 4. Comparison of the simulated vs. observed backscattering coefficients $\left(\sigma^{\circ}\right)$, where the triangles and the dots denote the Gaofen-3 and Radarsat-2 SAR data, respectively.

\subsection{Contribution of Scattering Components to the Total Backscattering}

Quantifying the scattering contributions from individual wheat constituents is helpful for interpreting the backscattering mechanisms of the SAR responses to various wheat constituents. Therefore, the input data sets at the ripening stage are selected to analyse the contribution of scattering mechanisms. The wheat canopy height varies from $60 \mathrm{~cm}$ to $85 \mathrm{~cm}$, while each of other parameters is assigned a constant that calculated by averaging parameters measured at a total of 25 sites sampled on June 1, 2017. The radar system parameters are identical to those of Radarsat- 2 image used in this study, i.e. microwave frequency $(5.405 \mathrm{GHz})$, incident angle $\left(23.54^{\circ}\right)$ and polarization mode $(\mathrm{HH}, \mathrm{VH}$ and $\mathrm{VV})$. Driven by the inputs, total backscatter and scattering components such as volume scattering from wheat ears, leaves and stems, their interaction with ground (doublebounce scattering), in conjunction with surface scattering of ground, are consequently simulated by WCSM.

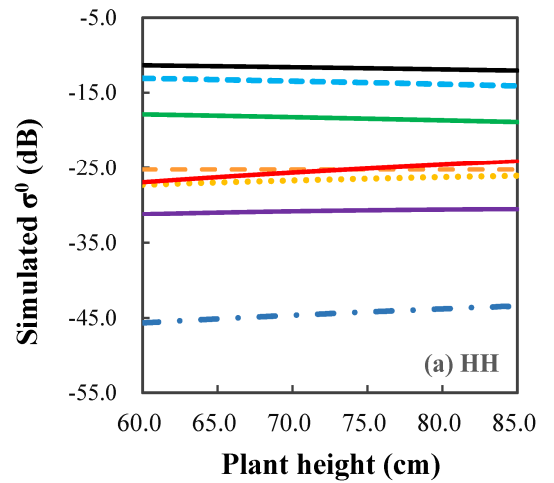

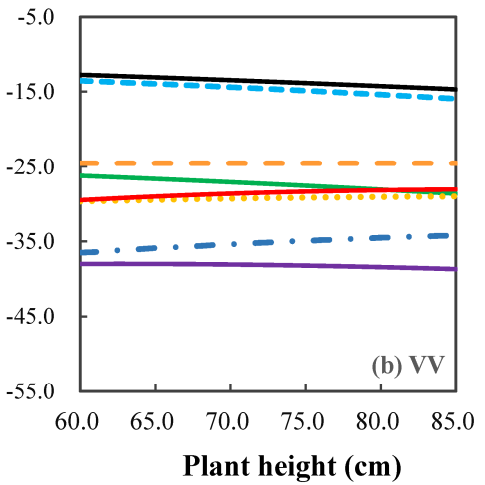

Plant height (cm)

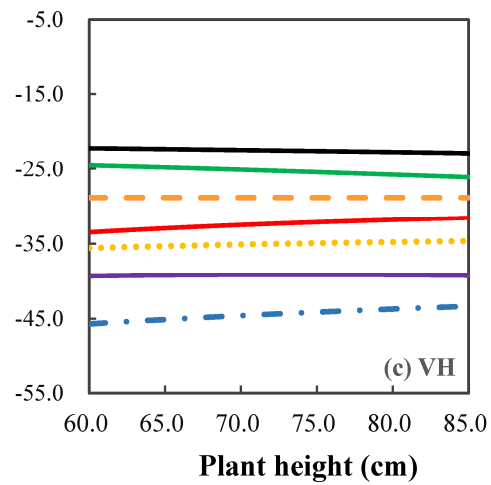

Plant height $(\mathbf{c m})$

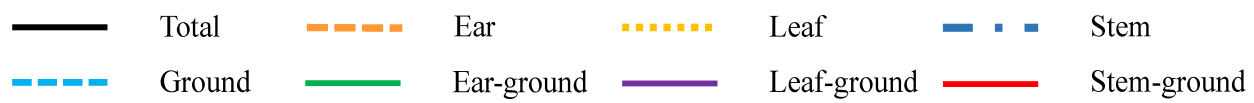

Figure 5. Contribution of different scattering mechanisms to the total backscatter of wheat canopy with varied plant height. 

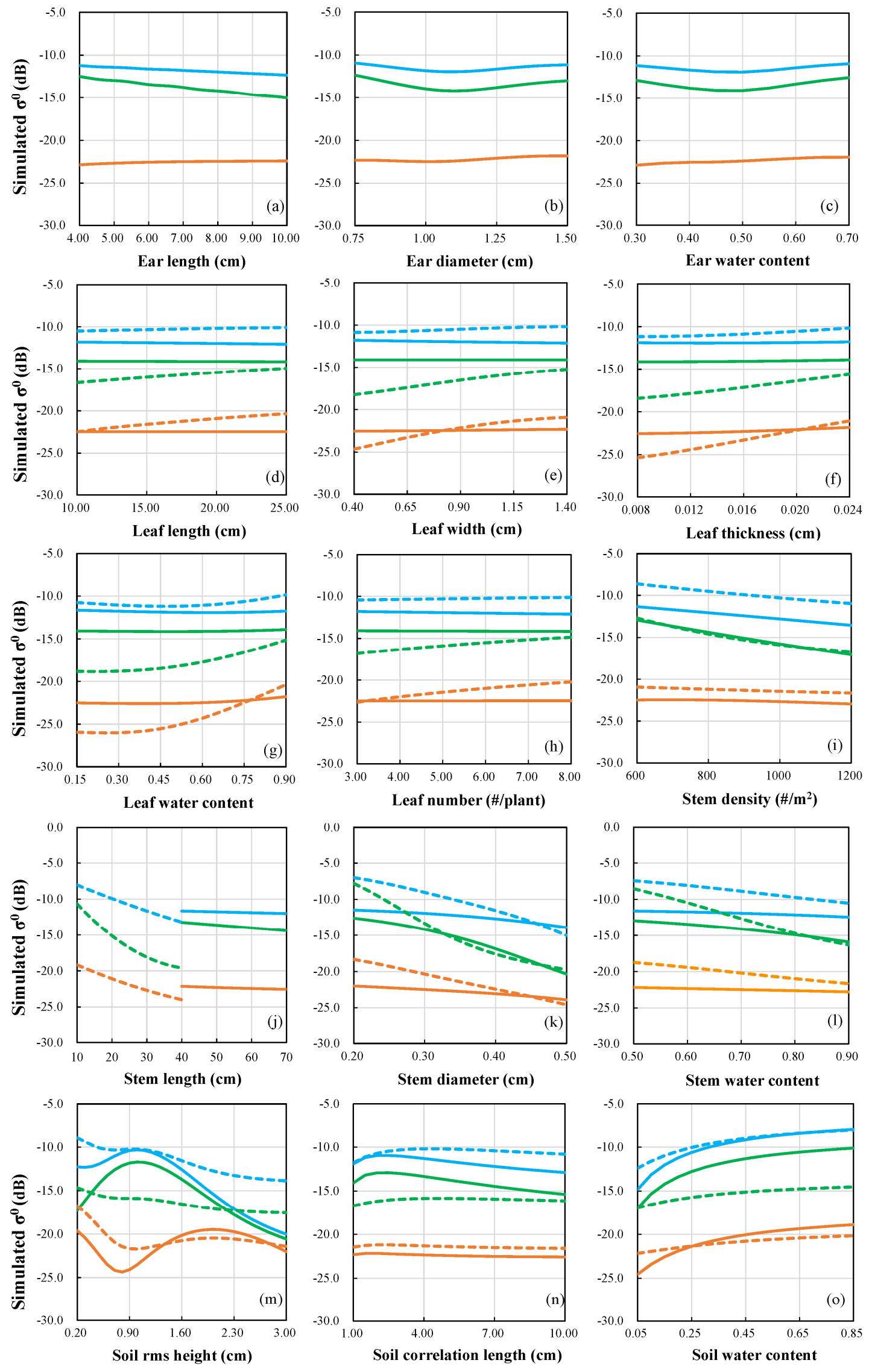

Figure 6. Sensitivity of physical parameters of wheat fields to total canopy backscatters. 
In agreement with surface scattering of underlying grounds, there is a slow decreasing trend in total scattering with increasing plant height. The direct ground backscattering mechanism make a significant contribution to total scattering for like-polarizations at low incident angle (Figures 5a, b), and the simulated results from WCSM are consistent with that reported in [23]. A possible reason for this is that the surface scattering is attenuated by the increasing size and number density of wheat plants. Ears are the particularly important constituents of wheat canopy. The canopy backscatters come largely from ear-ground double bounce which experiences a gradual drop resulting from the attenuation of the lower layer. Ear volume scattering relatively dominates in the total canopy backscatter, especially in VV polarization (Figure 5b). The leaf volume scattering contributes much more to the total canopy than its interactions with ground at all polarizations. In particular, leaf-ground scatter contributes the least in VV. As far as wheat stems are concerned, the scattering signals are governed predominantly by the stem-ground interactions. The stem-ground double bounce scattering in co-polarized backscatters increases dramatically and surpasses the stem volume scattering. In contrast, stem volume scattering has the least contribution to the total canopy scattering in $\mathrm{HH}$ and $\mathrm{VH}$ polarizations.

\subsection{Sensitivity of WCSM to Wheat Parameters}

In order to examine the total backscatter responses to varying physical parameters of wheat canopy, the developed model WCSM was implemented to simulate the C-band quad-polarized backscattering coefficients. Generally, the wheat canopy experienced two distinct growth stages during an entire growing season, i.e. before heading and after heading. The radar parameter configurations was in accordance with the Radarsat- 2 system in C-band at $23.54^{\circ}$ incident angle. The input data sets at the elongating stage (April 14) and ripening stage (June 1) was respectively collected for the sensitive analysis. The sensitivity test was conducted by varying a single input parameter in a predefined range while keeping all other input parameters constant. The model responses to a specific biophysical parameter were presented in Figure 6.

As far as wheat ears are concerned, the co-polarized scattering decrease continuously with the increasing ear length. In contrast, the cross-term $(\mathrm{VH})$ experiences a slight rising until the ear length reaches about $6 \mathrm{~cm}$, and then keeps stable (Figure 6a). As ears grow thicker, the backscatters for $\mathrm{HH}$ and VV polarization show a consistently declining trend and then a slow rising one (Figure 6b). The effects of ear water content on wheat canopy scattering for co-polarized mode follow the same pattern as for ear diameter. The water content of $50 \%$ is a critical value for an evident opposite relationship between scattering coefficients and ear moisture (Figure 6c).

In the elongating stage, Leaf structural properties had notably positive effects on the scattering mechanism for $\mathrm{VV}$ and VH polarizations (Figures $6 \mathrm{~d}, \mathrm{~h}$ ). The HH backscatter has just a small dependence on leaf size (Figures 6d, f). However, all backscatters were insensitive to leaf at ripening stage, which could be attributed to the attenuation of ears and low leaf moisture content at the particular stage. With wheat canopy becoming denser, an evident decrease occurred for co-polar terms from elongating stage onwards, while a relatively insignificant variation for cross-polarizations (Figure 6i).

In general, simulated total backscatters gradually decreased with the growing wheat stems. In contrast to the ripening stage, the backscatters in all polarizations in wheat's vegetative phrase were more sensitive to the stem diameter. A remarkable feature of the stem scattering was the larger changing ranges in $\mathrm{VV}$ than other polarizations. The $\mathrm{VV}$ backscatter was less than the $\mathrm{HH}$ for both elongating and ripening stages (Figures $6 \mathrm{k}, 1$ ). This appeared to be attributed in part by the stronger attenuation of the wheat stems to the vertical polarized waves than the horizontal one [31].

All of backscatters varies rapidly with an increasing rms height of rough ground surfaces at the ripening stage, whilst for the no-ear stage a falling and fluctuating trend is observed in co-polarization terms and the cross-polarized one, respectively (Figure $6 \mathrm{~m}$ ). There is no evident variations in $\mathrm{VH}$ polarization with respect to the correlation length. Negligible impact on cross polarizations is observed with a wide range of correlation length (Figure 6n). Obviously, all co- and cross-polarization backscatters are positively related to the volumetric water content of soil (Figure 6o), especially in the case of the moisture of $<0.25$, which account for the soil moisture play a key role in the surface scattering [36]. Moreover, it can be found that the effect of soil moisture is higher at $\mathrm{HH}$ polarization than at $\mathrm{VV}$ and $\mathrm{HV}$ polarizations in the elongating stage (April 14), which is partly attributed to that vertical stems have more attenuation to $\mathrm{HH}$ than $\mathrm{VV}$ and HV polarized radar signals.

\section{Discussion}

This work developed a physical model for wheat canopy to simulate scattering characteristics with the changing canopy architecture from elongating to ripening stage. To achieve an effective simulation, the physical, geometric and dielectric properties of wheat canopy and ground surfaces were calculated in the WCSM precisely. Geometric orientation distribution of wheat constituents was accurately simulated for ears, leaves and stems, respectively. The percentage of leaf number in each of wheat layers was modified, and the factor $20 \%$ and $80 \%$ was allocated to the upper ear-leaf layer and the lower leaf-stem layer, respectively. In view of the small contribution of second-order scattering terms to the total backscatters of dense vegetation canopy [27, 37], the model was established on the basis of a first-order solution of radiative transfer equation.

Although ear scatterer was often simplified or neglected in past modelling studies, they are crucial contributors to Cband cross polarization $[21,32,39]$. In this study, ears in the 
WCSM were taken as important scatterers within the whole wheat canopy from heading to ripening stages (Figure 5). This could provide an insight to understand the scattering contributions of wheat ears. Moreover, physical properties of wheat constituents such as size, orientation distribution and water content were precisely quantified in the WCSM. The distributions of wheat scatterers were assumed to be uniform in past studies. Unlike the abovementioned, the polynomial curve was selected to represent the leaf orientation, while two cosine function curves was respectively selected for the wheat ear and stem. The precise numerical representation of the scatterer orientation performed reasonably well in simulation of wheat backscatter of all linear polarizations. It presented potential applications for retrieval of wheat ear parameters and further yield estimation with SAR data. In comparison with existing works based on ground-based scatterometer or airborne SAR data [29, 30, 39], the simulated backscattering coefficients of wheat canopy in this study were in consistency with those satellite observed.

Although a reasonable simulation in total accuracy was achieved, considerable differences between the simulated and the SAR observed backscattering coefficients were found at several exceptional sampling sites. This disagreement could be attributed to several possible reasons. Over-simple assumption on leaf morphology (straight elliptical disks) could result in some uncertainties to the modelling of wheat canopy scattering. Since the booting stage, wheat leaves began to bend and the stem becoming hollow [25, 40]. The assumption on straight elliptic disk for leaf and solid cylinder for stem in the WCSM remained through the growing season. Also, the WCSM employed the same PDF to simulate the orientation distribution of leaves for all growth stages, which might contribute to the total simulation errors. In addition, the measurement accuracy in physical parameters of wheat plants and soil surfaces might be a large error resource for the simulation inaccuracy. The scale differences between field size at sampling sites and spatial resolution $(10 \mathrm{~m} \times 10 \mathrm{~m})$ of SAR imagery inevitably introduced some errors to the simulation of total canopy backscatter. Additionally, for the two Gaofen-3 SAR data, their acquisition dates (April 23 and May 27) were not temporally synchronous with the fieldworks (April 26 and May 25). The discrepancy in observation dates would to some extent influence the assessment of the WCSM's simulation validity.

For some sites, the observed backscattering coefficients from SAR images were extraordinarily higher or lower than simulated results. Elaborate examination confirmed that the abnormalities mainly appeared in some small wheat fields where row direction was rightly perpendicular/parallel to the look direction of radar. This further confirmed that row pattern effects were much more sensitive to co-polarization than cross-polarization [41, 42]. Particularly, the prominent influence of row pattern was observed in the incident angle ranges of $25^{\circ}$ to $40^{\circ}$ [43], which were very popular for currently operating satellite radar systems. This illustrated that it was necessary to rectify the row-direction effect in advance when co-polarized SAR data were used for wheat parameters estimation and mapping. For cross-polarization data, the row direction influence was relatively insignificant. A reasonable explanation for the high errors was that random noises inherent in SAR images gave rise to abrupt increases or drops in backscattering intensity of radar signals [44]. Adapting a proper filtering algorithm could be an effective alternative for eliminating such adverse effect.

\section{Conclusion}

This work presented a wheat canopy scattering model (WCSM) based on a first-order microwave radiative transfer theory to interpret the C-band microwave radar responses of wheat fields at multiple growth stages. The simulated backscattering coefficients were in agreement with the observed ones from Radarsat-2 and Gaofen-3 SAR data with error of $<1.8 \mathrm{~dB}$. The proposed model reasonably accounted for the scattering mechanisms of wheat crops, which includes volume scattering of wheat constituents (ears leaves and stems) and their double-bounce scattering with ground surfaces, as well as surface scattering from the underlying soil surfaces. In addition, this study shows that row direction of wheat fields has a significant impact for the total backscatters, further improvement in the model will be conduct to quantify the relative azimuth angles of radar beams for eliminating the variability in total backscatters. How to quantify the physical and geometric features of wheat canopy parameters as accurate and precise as possible is still a challenge for an effective simulation. Generally, the WCSM was effective and efficient tool in characterizing the scattering characteristics of wheat canopies at linear quadpolarization modes $(\mathrm{HH}, \mathrm{HV} / \mathrm{VH}$ and $\mathrm{VV})$ and different incidence angles. Meanwhile, this study indicated the WCSM has a potential value for parameter retrieval of wheat plants and soil surfaces over a large area.

Current on-orbit radar satellites (e.g. Sentinel-1, Radarsat2 and Gaofen-3) and future constellations missions such as the Radarsat constellation will make the availability of Cband SAR data more flexible. Further work for wheat canopy scattering simulation is consequently required to better combine the time series SAR data with substantial groundtruth measurements. The WCSM integrating an optimization algorithm would become a useful tool to extend the applications of C-band SAR data for operational growth monitoring of wheat crops and further yield estimation.

\section{Acknowledgements}

This work was supported by the National Natural Science Foundation of China under Grant No. 41571410.

\section{References}

[1] Godfray, H. C. J., J. R. Beddington, I. R. Crute, L. Haddad, D. Lawrence, J. F. Muir, J. Pretty, S. Robinson, S. M. Thomas and C. Toulmin (2010). Food security: the challenge of feeding 9 billion people". Science 327, 812-818. 
[2] Woodhouse, I. H. (2006). Introduction to microwave remote sensing. Boca Raton: Taylor \& Francis.

[3] Liu, C., J. L. Shang, P. W. Vachon and H. Mcnairn (2013). Multiyear crop monitoring using polarimetric Radarsat-2 data. IEEE Transactions on Geoscience and Remote Sensing 51, $2227-2240$.

[4] Bargiel, D. (2017). A new method for crop classification combining time series of radar images and crop phenology information. Remote Sensing of Environment 198, 369-383.

[5] Song, Y. and J. Wang (2019). Mapping winter wheat planting area and monitoring its phenology using Sentinel-1 backscatter time series. Remote Sensing 11, 1-13.

[6] Hosseini, M. and H. Mcnairn (2017). Using multi-polarization C- and L-band synthetic aperture radar to estimate biomass and soil moisture of wheat fields. International Journal of Applied Earth Observation and Geoinformation 58, 50-64.

[7] Pichierri, M., I. Hajnsek, S. Zwieback and B. Rabus (2018). On the potential of polarimetric SAR interferometry to characterize the biomass, moisture and structure of agricultural crops at L-, C- and X-bands. Remote Sensing of Environment 204, 596-616.

[8] Wang, H. Q., R. Magagi and K. Goita (2018). Potential of a two-component polarimetric decomposition at C-band for soil moisture retrieval over agricultural fields. Remote Sensing of Environment 217, 38-51.

[9] Mcnairn, H. and B. Brisco (2004). The application of C-band polarimetric SAR for agriculture: a review. Canadian Journal of Remote Sensing, 30, 525-542.

[10] Macelloni, G., S. Paloscia, P. Pampaloni, F. Marliani and M. Gai (2001). The relationship between the backscattering coefficient and the biomass of narrow and broad leaf crops. IEEE Transactions on Geoscience and Remote Sensing 39, 873-884.

[11] Brown, S. C. M., S. Quegan, K. Morrison, J. C. Bennett and G Cookmartin (2003). High-resolution measurements of scattering in wheat canopies-implications for crop parameter retrieval. IEEE Transactions on Geoscience and Remote Sensing 41, 1602-1610.

[12] Mattia, F., T. Le Toan, G. Picard, F. I. Posa, A. D'Alessio, C. Notarnicola, A. M. Gatti, M. Rinaldi, G. Satalino and G. Pasquariello (2003). Multitemporal C-band radar measurements on wheat fields. IEEE Transactions on Geoscience and Remote Sensing 41, 1551-1560.

[13] Jia, M., L. Tong, Y. Zhang and Y. Chen (2013). Multitemporal radar backscattering measurement of wheat fields using multifrequency (L, S, C, and X) and fullpolarization. Radio Science 48, 471-481.

[14] Balenzano, A., F. Mattia, G. Satalino and M. W. J. Davidson (2011). Dense temporal series of C- and L-band SAR data for soil moisture retrieval over agricultural crops. IEEE Journal of Selected Topics in Applied Earth Observations and Remote Sensing 4, 439-450.

[15] Fontanelli, G., S. Paloscia, M. Zribi and A. Chahbi (2013). Sensitivity analysis of X-band SAR to wheat and barley leaf area index in the Merguellil Basin. Remote Sensing Letters 4, 1107-1116.

[16] Liao, C., J. Wang, J. Shang, X. Huang, J. Liu and T. Huffman
(2018). Sensitivity study of Radarsat-2 polarimetric SAR to crop height and fractional vegetation cover of corn and wheat. International Journal of Remote Sensing 39, 1475-1490.

[17] EI Hajj, M., N. Baghdadi, H. Bazzi and M. Zribi (2019). Penetration analysis of SAR signals in the $\mathrm{C}$ and $\mathrm{L}$ bands for wheat, maize, and grasslands. Remote Sensing 11, 1-14.

[18] Attema, E. P. W. and F. T. Ulaby (1978). Vegetation modeled as a water cloud. Radio Science 13, 357-364.

[19] Champion, I., L. Prevot and G. Guyot (2000). Generalized semi-empirical modelling of wheat radar response. International Journal of Remote Sensing 21, 1945-1951.

[20] Kweon, S. K. and Y. Oh (2015). A modified water-cloud model with leaf angle parameters for microwave backscattering from agricultural fields. IEEE Transactions on Geoscience and Remote Sensing 53, 2802-2809.

[21] He, L., L. Tong, Y. Li, Y. Chen, L. Tan and C. Guo (2016). Polarimetric analysis of radar backscatter from ground-based scatterometers and wheat biomass monitoring with advanced synthetic aperture radar images. Journal of Applied Remote Sensing 10, 026008.

[22] Han, D., H. Yang, C. Qiu, G. Yang, E. Chen, Y. Du, W. Yang and C. Zhou (2019). Estimating wheat biomass from GF-3 data and a polarized water cloud model. Remote Sensing Letters 10, 234-243.

[23] Toure, A., K. P. B. Thomson, G. Edwards, R. J. Brown and B. G. Brisco (1994). Adaptation of the MIMICS backscattering model to the agricultural context-wheat and canola at L and C bands. IEEE Transactions on Geoscience and Remote Sensing $32,47-61$.

[24] Bracaglia, M., P. Ferrazzoli and L. Guerriero (1995). A fully polarimetric multiple scattering model for crops. Remote Sensing of Environment 54, 170-179.

[25] Della Vecchia, A., L. Guerriero, I. Bruni and P. Ferrazzoli (2006). Hollow cylinder microwave model for stems. Journal of Electromagnetic Waves and Applications 20, 301-318.

[26] Karam, M. A. and A. K. Fung (1988). Electromagnetic scattering from a layer of finite length, randomly oriented, dielectric, circular cylinders over a rough interface with application to vegetation. International Journal of Remote Sensing 9, 1109-1134.

[27] Cookmartin, G., P. Saich, S. Quegan, R. Cordey, P. BurgessAllen and A. Sowter (2002). Modeling microwave interactions with crops and comparison with ERS-2 SAR observations. IEEE Transactions on Geoscience and Remote Sensing 38, 658-670.

[28] Stiles, J. M. and K. Sarabandi (2000). Electromagnetic scattering from grassland. I. a fully phase-coherent scattering model. IEEE Transactions on Geoscience and Remote Sensing 38, 339-348.

[29] Stiles, J. M., K. Sarabandi and F. T. Ulaby (2000). Electromagnetic scattering from grassland. II. Measurement and modeling results. IEEE Transactions on Geoscience and Remote Sensing 38, 349-356.

[30] Marliani, F., S. Paloscia, P. Pampaloni and J. A. Kong (2002). Simulating coherent backscattering from crops during the growing cycle. IEEE Transactions on Geoscience and Remote Sensing 40, 162-177. 
[31] Picard, G. and T. Le Toan (2002). A multiple scattering model for C-band backscatter of wheat canopies. Journal of Electromagnetic Waves and Applications 16, 1447-1466.

[32] Picard, G., T. Le Toan and F. Mattia (2003). Understanding C-band radar backscatter from wheat canopy using a multiplescattering coherent model. IEEE Transactions on Geoscience and Remote Sensing 41, 1583-1591.

[33] Yan, W., B. Yang and Y. Zhang (2018). Characterizing the Cband backscattering of winter-wheat canopy with a microwave radiative transfer model. In Proceedings of the 7th International Conference on Agro-geoinformatics (Agrogeoinformatics 2018), Hangzhou, China, 6-9 August 2018, pp. 439-444.

[34] Ulaby, F. T. and M. A. El-Rayes (1987). Microwave dielectric spectrum of vegetation - part II: dual-dispersion model. IEEE Transactions on Geoscience and Remote Sensing GE-25, 550557.

[35] Dobson, M. C. (1985). Microwave dielectric behavior of wet soil-part II: dielectric-mixing models. IEEE Transactions on Geoscience and Remote Sensing GE-23, 35-46.

[36] Chen, K. S., T. D. Wu, L. Tsang and Q. Li (2003). Emission of rough surfaces calculated by the integral equation method with comparison to three-dimensional moment method simulations. IEEE Transactions on Geoscience and Remote Sensing 41, 90-101.

[37] Karam, M. A., A. K. Fung, R. H. Lang and N. S. Chauhan (1992). A microwave scattering model for layered vegetation. IEEE Transactions on Geoscience and Remote Sensing 30, 767-784.
[38] Karam, M. A. and A. K. Fung (1989). Leaf-shape effects in electromagnetic wave scattering from vegetation. IEEE Transactions on Geoscience and Remote Sensing 27, 687-697.

[39] Huang, B., Y. Chen, L. He, L. Tong and Y. Wang (2015). Backscattering modeling of wheat using vector radiative transfer theory. Journal of Applied Remote Sensing 9, 097093.

[40] Della Vecchia, A., P. Ferrazzoli and L. Guerriero (2004). Modelling microwave scattering from long curved leaves. Waves in Random Media 14, S333-S343.

[41] Wegmüller, U., M. Santoro, F. Mattia, A. Balenzano, G. Satalino, P. Marzahn, G. Fischer, R. Ludwig and N. Floury (2011). Progress in the understanding of narrow directional microwave scattering of agricultural fields. Remote Sensing of Environment 115, 2423-2433.

[42] Zribi, M., O. Taconet, V. Ciarletti and D. Vidal-Madjar (2002). Effect of row structures on radar microwave measurements over soil surface. International Journal of Remote Sensing 23, 5211-5224.

[43] Beaudoin, A., T. Le Toan and Q. H. J. Gwyn (1990). SAR observations and modeling of the C-band backscatter variability due to multiscale geometry and soil moisture. IEEE Transactions on Geoscience and Remote Sensing 28, 886-895.

[44] Argenti, F., A. Lapini, T. Bianchi and L. Alparone (2013). A tutorial on speckle reduction in synthetic aperture radar images. IEEE Transactions on Geoscience and Remote Sensing 1, 6-35. 